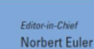

.

\section{Journal of Nonlinear Mathematical} Physics

ISSN (Online): 1776-0852 ISSN (Print): 1402-9251

Journal Home Page: https://www.atlantis-press.com/journals/inmp

\title{
Functional representation of the negative DNLS hierarchy
}

V.E. Vekslerchik

To cite this article: V.E. Vekslerchik (2013) Functional representation of the negative DNLS hierarchy, Journal of Nonlinear Mathematical Physics 20:4, 495-513, DOI: https://doi.org/10.1080/14029251.2013.868263

To link to this article: https://doi.org/10.1080/14029251.2013.868263

Published online: 04 January 2021 


\title{
Functional representation of the negative DNLS hierarchy
}

\author{
V.E. Vekslerchik \\ Usikov Institute for Radiophysics and Electronics \\ 12, Proskura st., Kharkov, 61085, Ukraine \\ vekslerchik@yahoo.com
}

Received 28 July 2013

Accepted 24 September 2013

\begin{abstract}
This paper is devoted to the negative flows of the derivative nonlinear Schrödinger hierarchy (DNLSH). The main result of this work is the functional representation of the extended DNLSH, composed of both positive (classical) and negative flows. We derive a finite set of functional equations, constructed by means of the Miwa's shifts, which contains all equations of the hierarchy. Using the obtained functional representation we convert the nonlocal equations of the negative subhierarchy into local ones of higher order, derive the generating function of the conservation laws and the N-soliton solutions for the extended DNLSH under non-vanishing boundary conditions.
\end{abstract}

Keywords: derivative NLS hierarchy, negative flows, functional representation, Miwa's shifts, conservation laws, solitons

2010 Mathematics Subject Classification: 37J35, 35Q51, 35Q55, 37K10

\section{Introduction.}

This paper, which can be viewed as a continuation of [22,23], is devoted to the negative flows of the derivative nonlinear Schrödinger (DNLS) hierarchy (DNLSH) [2,7,9]. Of three known forms of the DNLS equation (the Kaup-Newell [9], Chen-Lee-Liu [2] and Gerdjikov-Ivanov [7] equations) we use as the starting point the Chen-Lee-Liu equation,

$$
i U_{t}+U_{x x} \pm 2 i|U|^{2} U_{x}=0,
$$

which we write as a system

$$
\begin{aligned}
i U_{t}+U_{x x}+2 i U V U_{x} & =0 \\
-i V_{t}+V_{x x}+2 i U V V_{x} & =0
\end{aligned}
$$


Consequently, we use the term DNLSH for the hierarchy whose simplest equations are (1.2) (see the recent paper [4] on the negative flows of the Kaup-Newell hierarchy).

In this work we address several problems. First, we want to describe the extended DNLSH composed of both positive and negative flows and our main result is the functional representation of the positive, negative and mixed DNLS subhierarchies (sections 3-5). We derive a finite set of functional equations, constructed by means of the Miwa's shifts, which contains all equations of the extended hierarchy, that can be recovered by the power series expansion.

Considering various limiting cases of the obtained equations we demonstrate in section 5.1 that the extended DNLSH contains except the massive Thirring model (which is already known fact) such systems as Mikhailov-Fokas-Lenells equation, $(1+2)$-dimensional versions of the Chen-LeeLiu and Kaup-Newell equations as well as a 2-dimensional elliptic system with Toda-like nonlinearity which differs, apparently, from the DNLS-like systems.

The second goal of this paper is to derive the generating function for the conservation laws, which is presented in section 6. Contrary to the approach based on the inverse scattering transform, where such functions are expressed in terms of the scattering data and Jost functions, we formulate all results explicitly, in terms of solutions of the DNLSH.

Finally, we obtain in sections 7 and 8 the $N$-soliton solutions for the extended DNLSH under non-vanishing boundary conditions. Here we use another advantage of the functional representation and demonstrate how this problem can be solved by elementary algebraic calculations.

\section{Holonomy representation of the extended DNLSH.}

Instead of the so-called zero-curvature representation (ZCR) that is based on presenting the equations as the compatibility conditions for the linear differential systems

$$
\frac{\partial}{\partial t_{j}} \Psi=M_{j} \Psi, \quad \frac{\partial}{\partial \bar{t}_{k}} \Psi=\widetilde{M}_{k} \Psi, \quad j, k=1,2, \ldots
$$

we deal with the linear systems constructed by the Miwa's shift operators that are applied to functions of a doubly infinite number of arguments,

$$
U(\mathrm{t}, \overline{\mathrm{t}})=U\left(t_{1}, t_{2}, \ldots, \bar{t}_{1}, \bar{t}_{2}, \ldots\right)=U\left(t_{j}, \bar{t}_{k}\right)_{j, k=1,2, \ldots},
$$

and are defined by

$$
\begin{aligned}
& \mathbb{T}_{\xi} U(\mathrm{t}, \overline{\mathrm{t}})=U(\mathrm{t}+i[\xi], \overline{\mathrm{t}}) \\
& \overline{\mathbb{T}}_{\eta} U(\mathrm{t}, \overline{\mathrm{t}})=U(\mathrm{t}, \overline{\mathrm{t}}+i[\eta])
\end{aligned}
$$

or

$$
\begin{aligned}
& \mathbb{T}_{\xi} U\left(t_{j}, \bar{t}_{k}\right)_{j, k=1,2, \ldots}=U\left(t_{j}+i \xi^{j} / j, \bar{t}_{k}\right)_{j, k=1,2, \ldots} \\
& \overline{\mathbb{T}}_{\eta} U\left(t_{j}, \bar{t}_{k}\right)_{j, k=1,2, \ldots}=U\left(t_{j}, \bar{t}_{k}+i \eta^{k} / k\right)_{j, k=1,2, \ldots}
\end{aligned}
$$

Thus, our goal is to study the compatibility conditions of the systems of the following type:

$$
\begin{aligned}
\mathbb{T}_{\xi} \psi & =\mathrm{L}(\xi) \psi \\
\overline{\mathbb{T}}_{\eta} \psi & =\overline{\mathrm{L}}(\eta) \psi
\end{aligned}
$$


(where $\psi$ is a 2-vector, $L$ and $\bar{L}$ are $2 \times 2$-matrices) that are given by

$$
\begin{aligned}
{\left[\mathbb{T}_{\xi_{1}} \mathrm{~L}\left(\xi_{2}\right)\right] \mathrm{L}\left(\xi_{1}\right) } & =\left[\mathbb{T}_{\xi_{2}} \mathrm{~L}\left(\xi_{1}\right)\right] \mathrm{L}\left(\xi_{2}\right), \\
{\left[\mathbb{T}_{\xi} \mathrm{L}(\eta)\right] \mathrm{L}(\xi) } & =\left[\overline{\mathbb{T}}_{\eta} \mathrm{L}(\xi)\right] \overline{\mathrm{L}}(\eta), \\
{\left[\overline{\mathbb{T}}_{\eta_{1}} \overline{\mathrm{L}}\left(\eta_{2}\right)\right] \overline{\mathrm{L}}\left(\eta_{1}\right) } & =\left[\overline{\mathbb{T}}_{\eta_{2}} \mathrm{~L}\left(\eta_{1}\right)\right] \overline{\mathrm{L}}\left(\eta_{2}\right) .
\end{aligned}
$$

Using the standard strategy of the ZCR, that consists in introducing an auxiliary parameter, $\zeta$, and looking for the matrices $L$ and $\bar{L}$ with simplest dependence on $\zeta$, one can obtain the matrices that lead to the DNLSH. It turns out that $L$ and $\bar{L}$ should be linear functions of $\zeta$ and $\zeta^{-1}$ (see [23] for explanation). Omitting the details of the calculations we present here the 'minimal' solution for (2.6):

$$
\mathrm{L}(\xi)=\mathrm{L}(\xi ; \zeta)=\frac{1}{G(\xi)}\left(\begin{array}{cc}
\zeta \xi+G(\xi) & \xi V \\
-\zeta \xi \mathbb{T}_{\xi} U & 1
\end{array}\right)
$$

and

$$
\overline{\mathrm{L}}(\eta)=\overline{\mathrm{L}}(\eta ; \zeta)=\left(\begin{array}{cc}
\zeta^{-1} \eta+g(\eta) & \zeta^{-1} \eta v \\
-\eta \overline{\mathbb{T}}_{\eta} u & 1
\end{array}\right)
$$

where

$$
\begin{aligned}
& G(\xi)=1+\xi\left(\mathbb{T}_{\xi} U\right) V \\
& g(\eta)=1+\eta\left(\overline{\mathbb{T}}_{\eta} u\right) v .
\end{aligned}
$$

The functions $U, V, u$ and $v$ are subjected to the constraints that are discussed below.

In the following sections we study the systems of equations (the subhierarchies of the extended DNLSH) that appear as the result of (2.6) combined with (2.7) and (2.8).

\section{Positive DNLS subhierarchy.}

The equations that follow from (2.6a) are

$$
\begin{aligned}
& 0=\left(\xi_{1} G_{2}-\xi_{2} G_{1}\right)\left(\mathbb{T}_{12} U\right)-\xi_{1}\left(\mathbb{T}_{1} U\right)+\xi_{2}\left(\mathbb{T}_{2} U\right) \\
& 0=\left[\xi_{1}\left(\mathbb{T}_{1} G_{2}\right)-\xi_{2}\left(\mathbb{T}_{2} G_{1}\right)\right] V-\xi_{1}\left(\mathbb{T}_{2} V\right)+\xi_{2}\left(\mathbb{T}_{1} V\right)
\end{aligned}
$$

where we use the simplified notation

$$
\mathbb{T}_{j}=\mathbb{T}_{\xi_{j}}, \quad G_{j}=G\left(\xi_{j}\right), \quad j=1,2 .
$$

By simple algebra one can verify that equations (3.1) ensure vanishing of all components of the matrix equation (2.6a). This system, that can be viewed as the functional representation of the positive DNLSH, has been derived in $[3,21]$.

Equations (3.1) can be viewed as 'basic' representation from which one can obtain a few different systems that are more appropriate for tackling different problems. 
V.E. Vekslerchik

For example, sending $\xi_{2}$ to zero one arrives at the system

$$
\begin{aligned}
i \xi \partial_{1} U & =\left[1+\xi U\left(\mathbb{S}_{\xi} V\right)\right]\left(U-\mathbb{S}_{\xi} U\right) \\
i \xi \partial_{1} V & =\left[1+\xi\left(\mathbb{T}_{\xi} U\right) V\right]\left(\mathbb{T}_{\xi} V-V\right)
\end{aligned}
$$

where

$$
\mathbb{S}=\mathbb{T}^{-1}
$$

and $\partial_{1}=\partial / \partial t_{1}$. From this representation it is easy to derive the 'individual' equations of the hierarchy. The simplest of them, which is the result of collecting the $\xi^{2}$-terms, is (1.2).

Another form of (3.1) can be obtained by introducing the operator $\partial(\xi)$ by

$$
\partial(\xi)=\sum_{j=1}^{\infty} \xi^{j} \partial_{j}
$$

Noting that

$$
\lim _{\xi_{1}, \xi_{2} \rightarrow \xi} \frac{1}{\xi_{1}-\xi_{2}}\left(\mathbb{T}_{\xi_{1}} \mathbb{T}_{\xi_{2}}^{-1}-1\right) f=i \xi^{-1} \partial(\xi) f
$$

one can rewrite (3.1) as

$$
\begin{aligned}
i \partial(\xi) U & =H(\xi)\left(\mathbb{T}_{\xi} U-U\right), \\
-i \partial(\xi) V & =H(\xi)\left(\mathbb{S}_{\xi} V-V\right)
\end{aligned}
$$

with

$$
H(\xi)=\left[1+\xi\left(\mathbb{T}_{\xi} U\right)\left(\mathbb{S}_{\xi} V\right)\right]^{-1} .
$$

We use this representation to derive the the generating function for the conservation laws (see section 6).

Bearing in mind the bilinearization that we need to obtain explicit solutions for the DNLSH it is convenient to introduce the 'potential' $\Psi$. Noting that

$$
\left[\mathbb{T}_{\xi_{2}} G\left(\xi_{1}\right)\right] G\left(\xi_{2}\right)-G\left(\xi_{1}\right)\left[\mathbb{T}_{\xi_{1}} G\left(\xi_{2}\right)\right]=0
$$

(this identity can be proved by straightforward application of (3.1)) one can present $G(\xi)$ as

$$
G(\xi)=\Phi\left(\mathbb{T}_{\xi} \Psi\right)
$$

where

$$
\Phi=\Psi^{-1}
$$

and rewrite (3.1) as follows:

$$
\begin{aligned}
& \xi_{1}^{-1}\left(1-\mathbb{S}_{1}\right)\left(\mathbb{S}_{2} \Psi\right) U=\xi_{2}^{-1}\left(1-\mathbb{S}_{2}\right)\left(\mathbb{S}_{1} \Psi\right) U \\
& \xi_{1}^{-1}\left(1-\mathbb{T}_{1}\right)\left(\mathbb{T}_{2} \Phi\right) V=\xi_{2}^{-1}\left(1-\mathbb{T}_{2}\right)\left(\mathbb{T}_{1} \Phi\right) V
\end{aligned}
$$

The positive DNLS subhierarchy, to repeat, is the classical DNLS hierarchy, that has been introduced in the 70's and which is one of the most well-studied integrable systems. That is why we do not discuss equations (3.1) and their consequences here in detail. 


\section{Negative DNLS subhierarchy.}

Equations that follow from the commutativity condition (2.6c) can be written as

$$
\begin{aligned}
& 0=\left(\eta_{1} g_{2}-\eta_{2} g_{1}\right)\left(\overline{\mathbb{T}}_{12} u\right)-\eta_{1}\left(\overline{\mathbb{T}}_{1} u\right)+\eta_{2}\left(\overline{\mathbb{T}}_{2} u\right), \\
& 0=\left(\eta_{1}\left(\overline{\mathbb{T}}_{1} g_{2}\right)-\eta_{2}\left(\overline{\mathbb{T}}_{2} g_{1}\right)\right) v-\eta_{1}\left(\overline{\mathbb{T}}_{2} v\right)+\eta_{2}\left(\overline{\mathbb{T}}_{1} v\right) .
\end{aligned}
$$

It is easy to see that these equations become nothing but equations (3.1) after the substitution

$$
u, v, \overline{\mathbb{T}} \rightarrow U, V, \mathbb{T}
$$

or, in other words, the 'purely negative' DNLS subhierarchy is identical to the 'classical' one. Thus, we do not repeat the consideration of the previous section and present here only the identity

$$
\left(\overline{\mathbb{T}}_{2} g_{1}\right) g_{2}-g_{1}\left(\overline{\mathbb{T}}_{1} g_{2}\right)=0
$$

which is used below.

\section{Mixed DNLS subhierarchy.}

In this section we study the main object of this paper, the mixed (or extended) DNLSH. The results of the previous section demonstrate that this hierarchy can be thinked of as a result of integrable coupling of two copies of the 'classical' DNLSH. Equations that are discussed below are closely related to the equations that usually appear in the works devoted to the negative flows of any hierarchy.

Substituting matrices (2.7) and (2.8) into (2.6b) one arrives at

$$
\begin{aligned}
g(\eta)\left(\overline{\mathbb{T}}_{\eta} U\right)-U & =\eta\left(\overline{\mathbb{T}}_{\eta} u\right) \\
g(\eta) V-\overline{\mathbb{T}}_{\eta} V & =\eta v \\
G(\xi)\left(\mathbb{T}_{\xi} u\right)-u & =\xi\left(\mathbb{T}_{\xi} U\right) \\
G(\xi) v-\mathbb{T}_{\xi} v & =\xi V
\end{aligned}
$$

Using (3.10) and similar representation of $g(\eta)$,

$$
g(\eta)=\Psi\left(\overline{\mathbb{T}}_{\eta} \Phi\right)
$$

which follows from (4.3) and the identity

$$
\left[\mathbb{T}_{\xi} g(\eta)\right]\left[\overline{\mathbb{T}}_{\eta} G(\xi)\right]=g(\eta) G(\xi)
$$

stemming from (3.1), (4.1) and (5.1), one can eliminate from this system the 'negative variables' $u$ and $v$ coming to equations that can be written as

$$
\begin{aligned}
{\left[\mathbb{S}_{\xi}-\left(\mathbb{S}_{\xi} \Phi\right) \Psi\right]\left[\overline{\mathbb{S}}_{\eta}-\Phi\left(\overline{\mathbb{S}}_{\eta} \Psi\right)\right] U } & =\xi \eta U \\
{\left[\mathbb{T}_{\xi}-\Phi\left(\mathbb{T}_{\xi} \Psi\right)\right]\left[\overline{\mathbb{T}}_{\eta}-\left(\overline{\mathbb{T}}_{\eta} \Phi\right) \Psi\right] V } & =\xi \eta V
\end{aligned}
$$

Namely this system may be viewed as the mixed, or extended, DNLSH because it is written for the functions $U$ and $V$ (for which the equations of the positive DNLSH are written) and describes both 
positive and negative flows. It is still non-local because of the presence of $\Psi$, but can be made such, if considered together with, e.g. (3.10),

$$
\mathbb{T}_{\xi} \Psi=\left[1+\xi\left(\mathbb{T}_{\xi} U\right) V\right] \Psi
$$

as a system for the triple $U, V$ and $\Psi$. However, it is more convenient to work with the 'first-order' system (5.1), which we rewrite now in a few alternative forms.

Passing from the Miwa shifts $\mathbb{T}_{\xi}$ and $\overline{\mathbb{T}}_{\eta}$ to the $\partial(\xi)$ - and $\bar{\partial}(\eta)$-operators, where $\partial(\xi)$ is defined by (3.5) and

$$
\bar{\partial}(\eta)=\sum_{k=1}^{\infty} \eta^{k} \bar{\partial}_{k}, \quad \bar{\partial}_{k}=\partial / \partial \bar{t}_{k},
$$

one can obtain

$$
\begin{aligned}
i \bar{\partial}(\eta) U & =\eta h(\eta)\left[1-U\left(\overline{\mathbb{S}}_{\eta} v\right)\right]\left(\overline{\mathbb{T}}_{\eta} u\right) \\
-i \bar{\partial}(\eta) V & =\eta h(\eta)\left[1-\left(\overline{\mathbb{T}}_{\eta} u\right) V\right]\left(\overline{\mathbb{S}}_{\eta} v\right) \\
i \partial(\xi) u & =\xi H(\xi)\left[1-u\left(\mathbb{S}_{\xi} V\right)\right]\left(\mathbb{T}_{\xi} U\right) \\
-i \partial(\xi) v & =\xi H(\xi)\left[1-\left(\mathbb{T}_{\xi} U\right) v\right]\left(\mathbb{S}_{\xi} V\right)
\end{aligned}
$$

where $H(\xi)$ is given by (3.8) and

$$
h(\eta)=\left[1+\eta\left(\overline{\mathbb{T}}_{\eta} u\right)\left(\overline{\mathbb{S}}_{\eta} v\right)\right]^{-1}
$$

Calculating the derivatives of $G(\xi)$ and $g(\eta)$ and comparing the result with (3.10) and (5.2) one arrives at

$$
\begin{aligned}
H(\xi) & =1-i \partial(\xi) \ln \Psi \\
h(\eta) & =1+i \bar{\partial}(\eta) \ln \Psi
\end{aligned}
$$

which leads to, probably, the shortest form of the extended DNLSH:

$$
\begin{aligned}
i \bar{\partial}(\eta) \Phi U & =\eta h(\eta) \Phi\left(\overline{\mathbb{T}}_{\eta} u\right) \\
-i \bar{\partial}(\eta) \Psi V & =\eta h(\eta) \Psi\left(\overline{\mathbb{S}}_{\eta} v\right) \\
i \partial(\xi) \Psi u & =\xi H(\xi) \Psi\left(\mathbb{T}_{\xi} U\right) \\
-i \partial(\xi) \Phi v & =\xi H(\xi) \Phi\left(\mathbb{S}_{\xi} V\right)
\end{aligned}
$$

\subsection{Examples.}

Here we present a few simplest (and hence most representative) equations of the mixed DNLSH. 


\subsubsection{Example 1: Mikhailov-Fokas-Lenells equation.}

One can obtain from (5.10) in the $\xi, \eta \rightarrow 0$ limit that functions

$$
Q=\Phi U, \quad R=\Psi V
$$

considered as functions of

$$
x=t_{1}, \quad y=\bar{t}_{1}
$$

satisfy

$$
\begin{aligned}
& 0=Q_{x y}-2 i Q R Q_{y}+Q, \\
& 0=R_{x y}+2 i Q R R_{y}+R
\end{aligned}
$$

where the subscripts stand for the derivatives with respect to the corresponding variables. It is easy to see that these equations are the relativistically invariant two-dimensional field model studied by Mikhailov (see $[4,6,7]$ ) which has reappeared in the recent literature as the Fokas-Lenells system $[5,12,13]$.

\subsubsection{Example 2: $(1+2)$-dimensional Chen-Lee-Liu equation.}

Considering equations (5.7) or (5.10) in the $\xi \rightarrow 0$ limit one can obtain for the functions $Q$ and $R$ given by (5.11) and function $P(\eta)$,

$$
P(\eta)=h(\eta)-1
$$

the following system:

$$
\begin{aligned}
& 0=\bar{\partial}_{1} \bar{\partial}(\eta) Q+i \eta^{-1} \bar{\partial}(\eta) Q-i[1+2 P(\eta)] \bar{\partial}_{1} Q \\
& 0=\bar{\partial}_{1} \bar{\partial}(\eta) R-i \eta^{-1} \bar{\partial}(\eta) R+i[1+2 P(\eta)] \bar{\partial}_{1} R \\
& 0=\partial_{1} P(\eta)-\bar{\partial}(\eta) Q R
\end{aligned}
$$

which after expansion in power series in $\eta$ leads to

$$
\begin{aligned}
& 0=\bar{\partial}_{1} \bar{\partial}_{k} Q+i \bar{\partial}_{k+1} Q-2 i P^{(k)} \bar{\partial}_{1} Q, \\
& 0=\bar{\partial}_{1} \bar{\partial}_{k} R-i \bar{\partial}_{k+1} R+2 i P^{(k)} \bar{\partial}_{1} R, \\
& 0=\partial_{1} P^{(k)}-\bar{\partial}_{k} Q R
\end{aligned}
$$

(here, $P^{(k)}$ are the coefficients of the Taylor series for $P(\eta)$ ). The simplest equations of this hierarchy, rewritten in terms of variables $t, x$ and $y$,

$$
t=\bar{t}_{2}, \quad x=\bar{t}_{1}, \quad y=t_{1},
$$

are

$$
\begin{aligned}
& 0=i Q_{t}+Q_{x x}-2 i P Q_{x}, \\
& 0=-i R_{t}+R_{x x}+2 i P R_{x}, \\
& 0=P_{y}-(Q R)_{x}
\end{aligned}
$$

with $P=P^{(1)}$. It is easy to see that the reduction $x=y$ converts equations (5.18) into the Chen-LeeLiu equation (1.2), thus one can consider them as a $(1+2)$-dimensional version of the latter. 
V.E. Vekslerchik

5.1.3. Example 3: $(1+2)$-dimensional Kaup-Newell equation.

Proceeding as in the previous example, but choosing this time

$$
Q=\Psi U, \quad R=\Phi V
$$

with $P(\eta)$ being defined by (5.14), one can derive from (5.7)

$$
\begin{aligned}
& 0=\partial_{1}[\bar{\partial}(\eta)+2 i P(\eta)] Q+i \eta \bar{\partial}(\eta) Q+\eta Q, \\
& 0=\partial_{1}[\bar{\partial}(\eta)-2 i P(\eta)] R-i \eta \bar{\partial}(\eta) R+\eta R, \\
& 0=\partial_{1} P(\eta)-\bar{\partial}(\eta) Q R .
\end{aligned}
$$

The simplest of these equations (which come from collecting the $\eta^{2}$-terms) can be presented in terms of

$$
P=P^{(2)}, \quad t=\bar{t}_{1}, \quad x=t_{1}, \quad y=\bar{t}_{2}
$$

as the system

$$
\begin{aligned}
& 0=i Q_{t}+Q_{x y}+2 i(P Q)_{x}, \\
& 0=-i R_{t}+R_{x y}-2 i(P R)_{x}, \\
& 0=P_{x}-(Q R)_{y}
\end{aligned}
$$

that is nothing but a (1+2)-dimensional version of the Kaup-Newell equation [9].

\subsubsection{Example 4: Adler-Shabat $\mathrm{H}_{5}$ system.}

The appearance of Chen-Lee-Liu- and Kaup-Newell-like equations in the above examples is quite natural. However, from the extended DNLSH one can 'extract' some equations that are, at least seemingly, not of the DNLS-like type. One of them we want to present here.

It can be shown that equations (5.7) imply that the functions

$$
\lambda=\ln U, \quad \mu=\ln u
$$

satisfy

$$
\begin{aligned}
& 0=\partial_{1} \bar{\partial}_{1} \lambda-i e^{\mu-\lambda} \partial_{1} \lambda+i e^{\lambda-\mu} \bar{\partial}_{1} \lambda, \\
& 0=\partial_{1} \bar{\partial}_{1} \mu+i e^{\mu-\lambda} \partial_{1} \mu-i e^{\lambda-\mu} \bar{\partial}_{1} \mu
\end{aligned}
$$

which is (after the redefinition of the $t_{1}$ and $\bar{t}_{1}$ variables) the $H_{5}$ system from the classification of Adler and Shabat [1]. 


\subsubsection{Example 5: Massive Thirring model.}

Writing the simplest equations of (5.7)

$$
\begin{aligned}
i \bar{\partial}_{1} U & =-u v U+u, & i \partial_{1} u & =-U V u+U, \\
-i \bar{\partial}_{1} V & =-u v V+v, & -i \partial_{1} v & =-U V v+V
\end{aligned}
$$

or, in the case of the reduction $V=\mp U^{*}, v=\mp u^{*}$ where * stands for the complex conjugation,

$$
\begin{aligned}
i \bar{\partial}_{1} U & = \pm|u|^{2} U+u, \\
i \partial_{1} u & = \pm|U|^{2} u+U
\end{aligned}
$$

we reproduce results of $[4,6,7,17]$ on the relationship between the DNLSH and the massive Thirring model $[8,11,14,16]$.

One can generalize this proceedings by rewriting equations (5.7) in terms of the functions

$$
\begin{aligned}
& Q(\xi)=H(\xi)\left(\mathbb{T}_{\xi} U\right), \\
& R(\xi)=H(\xi)\left(\mathbb{S}_{\xi} V\right)
\end{aligned}
$$

and

$$
\begin{aligned}
& q(\eta)=h(\eta)\left(\overline{\mathbb{T}}_{\eta} u\right), \\
& r(\eta)=h(\eta)\left(\overline{\mathbb{S}}_{\eta} v\right) .
\end{aligned}
$$

Replacing $H(\xi)$ and $h(\eta)$ with

$$
K(\xi)=2 H(\xi)-1, \quad k(\eta)=2 h(\eta)-1
$$

which are related to $Q, R, q$ and $r$ by

$$
\begin{aligned}
K(\xi) & =\sqrt{1-4 \xi Q(\xi) R(\xi)}, \\
k(\eta) & =\sqrt{1-4 \eta q(\eta) r(\eta)}
\end{aligned}
$$

one can transform (5.7) into

$$
\begin{aligned}
i(1-\xi \eta) \bar{\partial}(\eta) Q(\xi) & =a(\xi, \eta) Q(\xi)+\eta K(\xi) q(\eta), \\
-i(1-\xi \eta) \bar{\partial}(\eta) R(\xi) & =a(\xi, \eta) R(\xi)+\eta K(\xi) r(\eta), \\
i(1-\xi \eta) \partial(\xi) q(\eta) & =A(\xi, \eta) q(\eta)+\xi k(\eta) Q(\xi), \\
-i(1-\xi \eta) \partial(\xi) r(\eta) & =A(\xi, \eta) r(\eta)+\xi k(\eta) R(\xi)
\end{aligned}
$$

where

$$
\begin{aligned}
& 2 A(\xi, \eta)=(1+\xi \eta) K(\xi)-1+\xi \eta, \\
& 2 a(\xi, \eta)=(1+\xi \eta) k(\eta)-1+\xi \eta .
\end{aligned}
$$

Equations (5.32), whose vector form is given by

$$
\begin{gathered}
i(1-\xi \eta) \sigma_{3} \bar{\partial}(\eta) \mathbf{Q}(\xi)=a(\xi, \eta) \mathbf{Q}(\xi)+\eta K(\xi) \mathbf{q}(\eta), \\
i(1-\xi \eta) \sigma_{3} \partial(\xi) \mathbf{q}(\eta)=A(\xi, \eta) \mathbf{q}(\eta)+\xi k(\eta) \mathbf{Q}(\xi)
\end{gathered}
$$


with

$$
\mathbf{Q}(\xi)=\left(\begin{array}{c}
Q(\xi) \\
R(\xi)
\end{array}\right), \quad \mathbf{q}(\eta)=\left(\begin{array}{l}
q(\eta) \\
r(\eta)
\end{array}\right), \quad \sigma_{3}=\left(\begin{array}{cc}
1 & 0 \\
0 & -1
\end{array}\right)
$$

can be viewed as the massive Thirring hierarchy.

\section{Constants of motion and conservation laws.}

The 'classical' (positive) DNLSH, as an integrable system, possesses an infinite number of constants of motion that can be presented as

$$
\mathscr{I}_{\ell}\left(t_{2}, t_{3}, \ldots\right)=\int \mathscr{J}_{\ell}\left(t_{1}, t_{2}, t_{3}, \ldots\right) d t_{1}, \quad \ell=0,1, \ldots
$$

Clearly, one can consider $\mathscr{I}_{\ell}$ as functions of a twice infinite set of variables

$$
\mathscr{I}_{\ell}=\mathscr{I}_{\ell}\left(t_{2}, t_{3}, \ldots, \bar{t}_{1}, \bar{t}_{2}, \ldots\right)
$$

being the constants with respect to both positive and negative 'times':

$$
\begin{array}{ll}
\partial \mathscr{I}_{\ell} / \partial t_{j}=0 & j=2,3, \ldots \\
\partial \mathscr{I}_{\ell} / \partial \bar{t}_{k}=0 & k=1,2, \ldots
\end{array}
$$

It turns out that the generating function for $\mathscr{J}_{\ell}$,

$$
\mathscr{J}(\zeta)=\sum_{\ell=0}^{\infty} \mathscr{J}_{\ell} \zeta^{\ell}
$$

has a very simple form when rewritten in terms of the Miwa's shifts:

$$
\mathscr{J}(\zeta)=\left(\mathbb{T}_{\zeta} U\right) V
$$

The main result of this section is given by the following

Proposition 6.1. The function $\mathscr{J}(\zeta)$ given by (6.5) satisfies equations

$$
\begin{aligned}
(\zeta-\xi) \partial(\xi) \mathscr{J}(\zeta) & =\xi \partial_{1} \mathscr{K}(\zeta, \xi) \\
(1-\zeta \eta) \bar{\partial}(\eta) \mathscr{J}(\zeta) & =\partial_{1} \mathscr{L}(\zeta, \eta)
\end{aligned}
$$

where

$$
\begin{aligned}
\mathscr{K}(\zeta, \xi) & =H(\xi)\left[1+\zeta\left(\mathbb{T}_{\zeta} U\right)\left(\mathbb{S}_{\xi} V\right)\right] \\
\mathscr{L}(\zeta, \eta) & =h(\eta)\left[1-\zeta \eta\left(\mathbb{T}_{\zeta} U\right)\left(\overline{\mathbb{S}}_{\eta} v\right)\right]
\end{aligned}
$$

whose proof is outlined in Appendix A. 
Expanding $\mathscr{K}(\zeta, \xi)$ and $\mathscr{L}(\zeta, \eta)$ in the double series,

$$
\begin{aligned}
\frac{\xi}{\zeta-\xi} \mathscr{K}(\zeta, \xi) & =\sum_{\ell=0}^{\infty} \sum_{j=1}^{\infty} \mathscr{K}_{\ell j} \zeta^{\ell} \xi^{j} \\
\frac{1}{1-\zeta \eta} \mathscr{L}(\zeta, \eta) & =1+\sum_{\ell=0}^{\infty} \sum_{k=1}^{\infty} \mathscr{L}_{\ell k} \zeta^{\ell} \eta^{k}
\end{aligned}
$$

one arrives at local conservation laws for all 'times', both positive and negative,

$$
\begin{aligned}
& \partial_{j} \mathscr{J}_{\ell}=\partial_{1} \mathscr{K}_{\ell j}, \\
& \bar{\partial}_{k} \mathscr{J}_{\ell}=\partial_{1} \mathscr{L}_{\ell k}
\end{aligned}
$$

for $\ell=0,1, \ldots$ and $j, k=1,2, \ldots$ with $t_{1}$ being the distinguished variable.

\section{Bilinearization.}

The first step of the bilinearization of the problem is to introduce the tau-functions, which can be done in a standard way:

$$
U=\frac{\sigma}{\tau}, \quad V=\frac{\tilde{\rho}}{\tilde{\tau}}
$$

and

$$
u=\frac{\tilde{\sigma}}{\tilde{\tau}}, \quad v=\frac{\rho}{\tau} .
$$

The second (and less trivial) step is to present the potential $\Psi$ as $\Psi=\tilde{\tau} / \tau$ which implies

$$
G(\xi)=\frac{\tau\left(\mathbb{T}_{\xi} \tilde{\tau}\right)}{\left(\mathbb{T}_{\xi} \tau\right) \tilde{\tau}}, \quad g(\eta)=\frac{\left(\overline{\mathbb{T}}_{\eta} \tau\right) \tilde{\tau}}{\tau\left(\overline{\mathbb{T}}_{\eta} \tilde{\tau}\right)}
$$

By a simple algebra one can show that equations (5.1) as well as definitions (2.9) and (2.10) become bilinear, which is demonstrated by the following

Proposition 7.1. The bilinear form of the extended DNLSH is given by the systems

$$
\begin{aligned}
& 0=\xi\left(\mathbb{T}_{\xi} \sigma\right) \tilde{\tau}-\left(\mathbb{T}_{\xi} \tilde{\sigma}\right) \tau+\tilde{\sigma}\left(\mathbb{T}_{\xi} \tau\right) \\
& 0=\xi \tilde{\rho}\left(\mathbb{T}_{\xi} \tau\right)-\rho\left(\mathbb{T}_{\xi} \tilde{\tau}\right)+\left(\mathbb{T}_{\xi} \rho\right) \tilde{\tau} \\
& 0=\xi \tilde{\rho}\left(\mathbb{T}_{\xi} \sigma\right)-\tau\left(\mathbb{T}_{\xi} \tilde{\tau}\right)+\left(\mathbb{T}_{\xi} \tau\right) \tilde{\tau}
\end{aligned}
$$

(the positive subhierarchy) and

$$
\begin{aligned}
& 0=\eta\left(\overline{\mathbb{T}}_{\eta} \tilde{\sigma}\right) \tau-\left(\overline{\mathbb{T}}_{\eta} \sigma\right) \tilde{\tau}+\sigma\left(\overline{\mathbb{T}}_{\eta} \tilde{\tau}\right) \\
& 0=\eta \rho\left(\overline{\mathbb{T}}_{\eta} \tilde{\tau}\right)-\tilde{\rho}\left(\overline{\mathbb{T}}_{\eta} \tau\right)+\left(\overline{\mathbb{T}}_{\eta} \tilde{\rho}\right) \tau \\
& 0=\eta \rho\left(\overline{\mathbb{T}}_{\eta} \tilde{\sigma}\right)-\left(\overline{\mathbb{T}}_{\eta} \tau\right) \tilde{\tau}+\tau\left(\overline{\mathbb{T}}_{\eta} \tilde{\tau}\right)
\end{aligned}
$$

(the negative one).

From the practical viewpoint, this form of the DNLSH is most suitable when one wants to derive explicit solutions. In section 8 we show how to obtain from (7.4) and (7.5) soliton solutions for 
the DNLSH by very simple calculations. However, for the sake of completeness, we show below the more traditional representation of the DNLSH in terms of the Hirota operators $D(\xi)$ and $\bar{D}(\eta)$ defined by

$$
D(\xi)=\sum_{j=1}^{\infty} \xi^{j} \bar{D}_{j}, \quad \bar{D}(\eta)=\sum_{k=1}^{\infty} \eta^{k} \bar{D}_{k}
$$

where

$$
\begin{aligned}
& D_{j} a \cdot b=\left(\partial_{j} a\right) b-a\left(\partial_{j} b\right), \\
& \bar{D}_{k} a \cdot b=\left(\bar{\partial}_{k} a\right) b-a\left(\bar{\partial}_{k} b\right)
\end{aligned}
$$

with $\partial_{j}=\partial / \partial t_{j}$ and $\bar{\partial}_{k}=\partial / \partial \bar{t}_{k}$. To do this, we need the two-shift generalizations of equations from Proposition 7.1, which we do not present here. Then, by taking the limits (as in (3.6)) one can obtain

$$
H(\xi)=c_{\xi} \frac{\left(\mathbb{T}_{\xi} \tau\right)\left(\mathbb{S}_{\xi} \tilde{\tau}\right)}{\tau \tilde{\tau}}, \quad h(\eta)=\bar{c}_{\eta} \frac{\left(\overline{\mathbb{S}}_{\eta} \tau\right)\left(\overline{\mathbb{T}}_{\eta} \tilde{\tau}\right)}{\tau \tilde{\tau}}
$$

(where $c_{\xi}$ and $\bar{c}_{\eta}$ are constants that depend on $\xi, \eta$ and the boundary conditions) and the following representation of the DNLSH:

Proposition 7.2. The Hirota-like form of the extended DNLSH is given by the systems

$$
\begin{aligned}
& i D(\xi) \tau \cdot \tilde{\tau}=-\tau \tilde{\tau}+c_{\xi}\left(\mathbb{T}_{\xi} \tau\right)\left(\mathbb{S}_{\xi} \tilde{\tau}\right) \\
& i D(\xi) \sigma \cdot \tilde{\tau}=-\sigma \tilde{\tau}+c_{\xi}\left(\mathbb{T}_{\xi} \sigma\right)\left(\mathbb{S}_{\xi} \tilde{\tau}\right) \\
& i D(\xi) \tilde{\rho} \cdot \tau=\tilde{\rho} \tau-c_{\xi}\left(\mathbb{S}_{\xi} \tilde{\rho}\right)\left(\mathbb{T}_{\xi} \tau\right) \\
& i D(\xi) \tilde{\sigma} \cdot \tau=c_{\xi} \xi\left(\mathbb{T}_{\xi} \sigma\right)\left(\mathbb{S}_{\xi} \tilde{\tau}\right) \\
& i D(\xi) \rho \cdot \tilde{\tau}=-c_{\xi} \xi\left(\mathbb{S}_{\xi} \tilde{\rho}\right)\left(\mathbb{T}_{\xi} \tau\right)
\end{aligned}
$$

(the positive subhierarchy) and

$$
\begin{aligned}
i \bar{D}(\eta) \tau \cdot \tilde{\tau} & =\tau \tilde{\tau}-\bar{c}_{\eta}\left(\overline{\mathbb{S}}_{\eta} \tau\right)\left(\overline{\mathbb{T}}_{\eta} \tilde{\tau}\right) \\
i \bar{D}(\eta) \tilde{\sigma} \cdot \tau & =-\tilde{\sigma} \tau+\bar{c}_{\eta}\left(\overline{\mathbb{T}}_{\eta} \tilde{\sigma}\right)\left(\overline{\mathbb{S}}_{\eta} \tau\right) \\
i \bar{D}(\eta) \rho \cdot \tilde{\tau}= & \rho \tilde{\tau}-\bar{c}_{\eta}\left(\overline{\mathbb{S}}_{\eta} \rho\right)\left(\overline{\mathbb{T}}_{\eta} \tilde{\tau}\right) \\
i \bar{D}(\eta) \sigma \cdot \tilde{\tau}= & \bar{c}_{\eta} \eta\left(\overline{\mathbb{T}}_{\eta} \tilde{\sigma}\right)\left(\overline{\mathbb{S}}_{\eta} \tau\right) \\
i \bar{D}(\eta) \tilde{\rho} \cdot \tau & =-\bar{c}_{\eta} \eta\left(\overline{\mathbb{S}}_{\eta} \rho\right)\left(\overline{\mathbb{T}}_{\eta} \tilde{\tau}\right)
\end{aligned}
$$

(the negative one).

\section{Solitons of the extended DNLSH under non-vanishing boundary conditions.}

In this section we present the soliton solutions for the extended (describing both positive and negative flows) DNLSH under non-vanishing boundary conditions. Calculations that lead to our goal are similar to ones described in [23]. Thus, here we only outline the main steps and present the main results. 


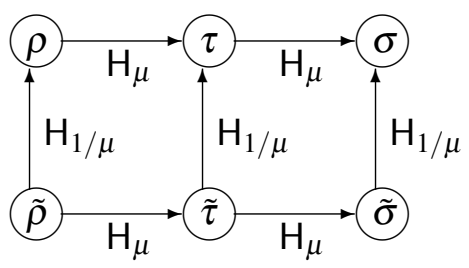

Fig. 1. Relations between the tau-functions.

The building blocks for the soliton solutions are $N \times N$ matrices A that satisfy the 'almost rankone' condition

$$
\mathrm{LA}-\mathrm{AL}^{-1}=|\ell\rangle\langle a|
$$

where $\mathrm{L}$ is a constant diagonal matrix, $|\ell\rangle$ is a constant $N$-component column, $|\ell\rangle=\left(\ell_{1}, \ldots, \ell_{N}\right)^{T}$, $\langle a|$ is a $N$-component row depending on the coordinates describing the DNLSH flows, $\langle a(\mathrm{t}, \overline{\mathrm{t}})|=$ $\left(a_{1}(\mathrm{t}, \overline{\mathrm{t}}), \ldots, a_{N}(\mathrm{t}, \overline{\mathrm{t}})\right)$, and matrices $\mathrm{H}_{\zeta}$ that are defined by

$$
\mathrm{H}_{\zeta}=(\zeta 1-\mathrm{L})\left(\zeta 1-\mathrm{L}^{-1}\right)^{-1}
$$

where 1 is the $N \times N$ unit matrix. The remarkable property of the above matrices, that is used below, is that the determinants

$$
\omega(\mathrm{A})=\operatorname{det}|1+\mathrm{A}|
$$

satisfy the Fay-like identity

$$
(\xi-\eta) \omega_{\zeta} \omega_{\xi \eta}+(\eta-\zeta) \omega_{\xi} \omega_{\eta \zeta}+(\zeta-\xi) \omega_{\eta} \omega_{\zeta \xi}=0
$$

where

$$
\omega=\omega(\mathrm{A}), \quad \omega_{\zeta}=\omega\left(\mathrm{AH}_{\zeta}\right), \quad \omega_{\xi \eta}=\omega\left(\mathrm{AH}_{\xi} \mathrm{H}_{\eta}\right) .
$$

To obtain the soliton solutions, first one needs to establish the relationships between six taufunctions. This can be done by means of matrices $\mathrm{H}_{\mu}$ and $\mathrm{H}_{1 / \mu}$, related by

$$
\mathrm{H}_{\mu} \mathrm{H}_{1 / \mu}=\mathrm{L}^{2}
$$

as follows (see Fig. 1):

$$
\tau=\tau_{*} \omega\left(\mathrm{AH}_{1 / \mu}\right), \quad \sigma=\sigma_{*} \omega\left(\mathrm{AH}_{\mu} \mathrm{H}_{1 / \mu}\right), \quad \rho=\rho_{*} \omega\left(\mathrm{AH}_{\mu}^{-1} \mathrm{H}_{1 / \mu}\right)
$$

and

$$
\tilde{\tau}=\tilde{\tau}_{*} \omega(\mathrm{A}), \quad \tilde{\sigma}=\tilde{\sigma}_{*} \omega\left(\mathrm{AH}_{\mu}\right), \quad \tilde{\rho}=\tilde{\rho}_{*} \omega\left(\mathrm{AH}_{\mu}^{-1}\right)
$$

The functions with the $*$-subscript (the background tau-functions) with simple transformation properties with respect to the Miwa shifts (they are eigenfunctions of $\mathbb{T}_{\xi}$ and $\overline{\mathbb{T}}_{\eta}$ ) are presented below. 
Secondly, one has to define the 'evolution' of the matrices A with respect to the Miwa shifts which, again, can be constructed by means of the $\mathrm{H}$-matrices,

$$
\mathbb{T}_{\xi} \mathrm{A}=\mathrm{AH}_{\alpha(\xi)} \mathrm{H}_{\alpha(0)}^{-1}, \quad \overline{\mathbb{T}}_{\eta} \mathrm{A}=\mathrm{AH}_{\beta(\eta)} \mathrm{H}_{\beta(0)}^{-1}
$$

where $\alpha(\xi)$ and $\beta(\eta)$ should be determined (see below).

Finally, one has to find the background tau-functions $\tau_{*}, \sigma_{*}, \rho_{*}, \tilde{\tau}_{*}, \tilde{\sigma}_{*}$ and $\tilde{\rho}_{*}$. This can be done by substituting (8.7) and (8.8) into the Fay identities and gathering all the constants. For example, equation (8.4) with $(\xi, \eta, \zeta)=(0,1 / \mu, \alpha)$ after the shift $\mathrm{A} \rightarrow \mathrm{AH}_{0}^{-1}$ and replacement of all $\omega\left(\mathrm{AH}_{\zeta}\right)$ with the corresponding tau-functions from (8.7), (8.8) and (8.9) becomes (under the assumption $\alpha(0)=0$ )

$$
\mu \alpha \frac{\tilde{\rho}}{\tilde{\rho}_{*}} \frac{\left(\mathbb{T}_{\xi} \sigma\right)}{\left(\mathbb{T}_{\xi} \sigma_{*}\right)}-\frac{\tau}{\tau_{*}} \frac{\left(\mathbb{T}_{\xi} \tilde{\tau}\right)}{\left(\mathbb{T}_{\xi} \tilde{\tau}_{*}\right)}+(1-\mu \alpha) \frac{\tilde{\tau}}{\tilde{\tau}_{*}} \frac{\left(\mathbb{T}_{\xi} \tau\right)}{\left(\mathbb{T}_{\xi} \tau_{*}\right)}=0 .
$$

Comparing this identity with $(7.4 \mathrm{c})$ one can conclude that to solve the latter one has to met the conditions

$$
\frac{1}{\xi} \frac{\mu \alpha(\xi)}{\tilde{\rho}_{*}\left(\mathbb{T}_{\xi} \sigma_{*}\right)}=\frac{1}{\tau_{*}\left(\mathbb{T}_{\xi} \tilde{\tau}_{*}\right)}=\frac{1-\mu \alpha(\xi)}{\tilde{\tau}_{*}\left(\mathbb{T}_{\xi} \tau_{*}\right)} .
$$

Repeating this proceeding with different choices of $(\xi, \eta, \zeta)$ one can reduce (7.4) to a system of equations similar to (8.11) that can be solved by the proper choice of the background tau-functions and $\alpha(\xi)$. This leads to the following results.

Proposition 8.1. Functions (8.7), (8.8) solve equations (7.4) provided the background tau-functions satisfy

$$
\begin{aligned}
& \tau_{*}^{2}=\left(1-\mu^{-2}\right) \sigma_{*} \rho_{*}, \\
& \tilde{\tau}_{*}^{2}=\left(1-\mu^{-2}\right) \tilde{\sigma}_{*} \tilde{\rho}_{*}
\end{aligned}
$$

while their dependence on positive times is governed by

$$
\begin{aligned}
& \left(\mathbb{T}_{\xi} \tau_{*}\right) \tilde{\tau}_{*}=\tau_{*}\left(\mathbb{T}_{\xi} \tilde{\tau}_{*}\right) \chi_{\tau}(\xi), \\
& \left(\mathbb{T}_{\xi} \sigma_{*}\right) \tau_{*}=\sigma_{*}\left(\mathbb{T}_{\xi} \tau_{*}\right) \chi_{\sigma}(\xi) \\
& \left(\mathbb{T}_{\xi} \tilde{\sigma}_{*}\right) \tilde{\tau}_{*}=\tilde{\sigma}_{*}\left(\mathbb{T}_{\xi} \tilde{\tau}_{*}\right) \chi_{\sigma}(\xi),
\end{aligned}
$$

where functions $\chi_{\tau}(\xi)$ and $\chi_{\sigma}(\xi)$ are given by

$$
\begin{aligned}
& \chi_{\tau}(\xi)=1-\mu \alpha(\xi), \\
& \chi_{\sigma}(\xi)=1-\mu^{-1} \alpha(\xi)
\end{aligned}
$$

and $\alpha(\xi)$ is the solution of

$$
\alpha(\xi)+\alpha(\xi)^{-1}=\mu+\mu^{-1}+c \mu \xi^{-1}, \quad \alpha(0)=0
$$

where constant $c$ determines the amplitude of the solutions,

$$
c=\frac{\tau_{*} \tilde{\tau}_{*}}{\sigma_{*} \tilde{\rho}_{*}}=\left(1-\mu^{-2}\right)^{2} \frac{\tilde{\sigma}_{*} \rho_{*}}{\tau_{*} \tilde{\tau}_{*}} .
$$

(The dependence of $\rho_{*}$ and $\tilde{\rho}_{*}$ on positive times, which is not written explicitly, can be recovered from (8.13) and (8.12).) 
Similar result can be obtained for the negative subhierarchy.

Proposition 8.2. Functions (8.7), (8.8) solve equations (7.4) provided the background tau-functions satisfy (8.12) while their dependence on negative times is governed by

$$
\begin{aligned}
& \left(\overline{\mathbb{T}}_{\eta} \tau_{*}\right) \tilde{\tau}_{*}=\tau_{*}\left(\overline{\mathbb{T}}_{\eta} \tilde{\tau}_{*}\right) \bar{\chi}_{\tau}(\eta), \\
& \left(\overline{\mathbb{T}}_{\eta} \sigma_{*}\right) \tau_{*}=\sigma_{*}\left(\overline{\mathbb{T}}_{\eta} \tau_{*}\right) \bar{\chi}_{\sigma}(\eta), \\
& \left(\overline{\mathbb{T}}_{\eta} \tilde{\sigma}_{*}\right) \tilde{\tau}_{*}=\tilde{\sigma}_{*}\left(\overline{\mathbb{T}}_{\eta} \tilde{\tau}_{*}\right) \bar{\chi}_{\sigma}(\eta)
\end{aligned}
$$

where functions $\bar{\chi}_{\tau}(\eta)$ and $\bar{\chi}_{\sigma}(\eta)$ are given by

$$
\begin{aligned}
& \bar{\chi}_{\tau}(\eta)=\frac{1-\mu \beta(\eta)}{1-\mu^{2}} \\
& \bar{\chi}_{\sigma}(\eta)=\frac{\beta(\eta)}{\mu} \frac{1-\mu^{2}}{1-\mu \beta(\eta)}
\end{aligned}
$$

and $\beta(\eta)$ is the solution of

$$
\beta(\eta)+\beta(\eta)^{-1}=\mu+\mu^{-1}+c \mu \eta, \quad \beta(0)=\mu .
$$

Knowing solutions for the bilinear system (7.4) and (7.5) it is easy to write down solutions for (5.1). Bearing in mind physical applications, one has to restrict himself with $U=\varepsilon V^{*}$ where the asterisk stands for the complex conjugation and $\varepsilon= \pm 1$. It should be noted that, contrary to the case of the nonlinear Schrödinger equation where the value of $\varepsilon$ is crucial (it determines, for example, the type of solitons, bright or dark), $\varepsilon$ can be eliminated from the DNLS equation by the substitution $U\left(t_{1}, t_{2}, \ldots\right) \rightarrow U\left(\varepsilon t_{1}, t_{2}, \ldots\right)$. Thus, we consider below only the case

$$
U=V^{*}
$$

This condition imposes some restrictions on the soliton matrices $\mathrm{A}$ and the transformation matrices $\mathrm{H}$. It can be shown that to resolve (8.20) one has to ensure the reality of $\mathrm{C}=\mathrm{AH}_{1 / \mu}^{1 / 2}, \mathrm{C}^{*}=\mathrm{C}$, and unitarity of all $\mathrm{H}$-matrices used above:

$$
\mathrm{H}_{\zeta} \mathrm{H}_{\zeta}^{*}=1, \quad \zeta=\mu, 1 / \mu, \alpha(\xi), \beta(\eta)
$$

for real $\xi$ and $\eta$. It is easy to verify that one can met these conditions by taking real $\mu$ and

$$
\mathrm{L}=\operatorname{diag}\left(e^{i \theta_{n}}\right)_{n=1, \ldots, N}
$$

which after omitted here calculations leads to

$$
\mathrm{C}(\mathrm{t}, \overline{\mathrm{t}})=\left(\mathrm{C}_{m n}^{(0)} e^{v_{n}(\mathrm{t}, \overline{\mathrm{t}})}\right)_{m, n=1, \ldots, N}
$$

where $\left(\mathrm{C}_{m n}^{(0)}\right)$ is a constant matrix and

$$
v_{n}(\mathrm{t}, \overline{\mathrm{t}})=\sum_{k=1}^{\infty}\left(v_{n k} t_{k}+\tilde{v}_{n k} \bar{t}_{k}\right)
$$


with

$$
\begin{aligned}
& \sum_{k=1}^{\infty} v_{n k} \xi^{k} / k=2 \arg \left[1-\alpha(\xi) e^{-i \theta_{n}}\right], \\
& \sum_{k=1}^{\infty} \tilde{v}_{n k} \eta^{k} / k=2 \arg \left[1-\beta(\eta) e^{-i \theta_{n}}\right]-2 \arg \left[1-\mu e^{-i \theta_{n}}\right] .
\end{aligned}
$$

The ratio of the background tau-functions, $U_{*}=\sigma_{*} / \tau_{*}$ can be presented as

$$
U_{*}=U^{(0)} e^{i \varphi(\mathrm{t}, \overline{\mathrm{t}})}
$$

with arbitrary constant $U^{(0)}$ that replaces $c$ in (8.15), (8.16), (8.19),

$$
c=\left|U^{(0)}\right|^{-2}
$$

and

$$
\varphi(\mathrm{t}, \overline{\mathrm{t}})=\sum_{k=1}^{\infty}\left(\varphi_{k} t_{k}+\tilde{\varphi}_{k} \bar{t}_{k}\right)
$$

where

$$
\begin{aligned}
& \sum_{k=1}^{\infty} \varphi_{k} \xi^{k} / k=-\ln \left[1-\mu^{-1} \alpha(\xi)\right] \\
& \sum_{k=1}^{\infty} \tilde{\varphi}_{k} \eta^{k} / k=\ln \left[1-\mu^{-1} \beta(\eta)^{-1}\right]-\ln \left[1-\mu^{-2}\right] .
\end{aligned}
$$

Finally, presenting $\mathrm{H}_{\mu} \mathrm{H}_{1 / \mu}^{1 / 2}$ and $\mathrm{H}_{1 / \mu}^{1 / 2}$ as $\operatorname{diag}\left(e^{i \gamma_{n}^{(1,2)}}\right)$ where

$$
\gamma_{n}^{(2)}=\arg \left(1-\mu e^{i \theta_{n}}\right), \quad \gamma_{n}^{(1)}=2 \theta_{n}-\gamma_{n}^{(2)},
$$

using (8.1) to obtain $C_{m n}^{(0)}$ and eliminating, without loss of generality, superfluous constants upon noting that the determinants (8.3) are invariant under transformations $A \rightarrow M^{-1} A M$, one arrives at the final expressions for the soliton solutions of the extended DNLSH:

Proposition 8.3. The $N$-soliton solutions for the DNLSH under non-vanishing boundary conditions can be presented as

$$
U(\mathrm{t}, \overline{\mathrm{t}})=U^{(0)} \exp [i \varphi(\mathrm{t}, \overline{\mathrm{t}})] \frac{\Delta_{1}(\mathrm{t}, \overline{\mathrm{t}})}{\Delta_{2}(\mathrm{t}, \overline{\mathrm{t}})}
$$

with arbitrary $U^{(0)}$ and

$$
\Delta_{\ell}(\mathrm{t}, \overline{\mathrm{t}})=\operatorname{det}\left|\delta_{m n}+C_{n}^{(0)} \frac{\exp \left[v_{n}(\mathrm{t}, \overline{\mathrm{t}})+i \gamma_{n}^{(\ell)}\right]}{\sin \left(\frac{\theta_{m}+\theta_{n}}{2}\right)}\right|_{m, n=1, \ldots, N} \quad(\ell=1,2)
$$

where functions $v(\mathrm{t}, \overline{\mathrm{t}})$ and $\varphi(\mathrm{t}, \overline{\mathrm{t}})$ are defined in (8.24), (8.25) and (8.28)-(8.30), $C_{n}^{(0)}$ are arbitrary real constants and $V=U^{*}$. 


\section{Conclusion.}

To conclude, we would like to summarize the main results of this paper and to outline possible continuations of the presented work.

The main subject of this paper is the extended DNLSH. The main results we have obtained are 1) the functional representation of both positive (classical) and negative flows, 2) the generating function for the conservation laws and 3) the dark-soliton solutions.

The most straightforward continuation of this work is to use the advantages of the functional representation and to derive other classes of explicit solutions which complement the bright-soliton solutions derived in [4] and dark solitons presented above. This can be done starting from the bilinear equations of proposition 7.1 which can be associated with the Fay identities for the thetafunctions and used to derive the quasiperiodic solutions, or with various determinant identities that lead to Wronskian, Toeplitz and other solutions.

Another range of arising problems is related to the results of section 5. We would like to stress that this paper is not aimed to elaborate methods of generating new integrable models, but the examples presented in section 5.1, which surely do not exhaust all models 'hidden' inside the DNLSH, demonstrate that the question of what systems can be obtained from (or reduced to) the equations of a given hierarchy (the DNLSH in our case) is far from trivial. Even in the short list of section 5.1 one can find a few 'new', i.e. not well-studied, ones: (1+2)-dimensional Chen-Lee-Liu equation (5.18), (1+2)-dimensional Kaup-Newell equation (5.22), Adler-Shabat $H_{5}$ system (5.24).

Considering the last one, it was obtained by Adler and Shabat in the framework of the classification of two-component hyperbolic systems. However, the result of [1] seems to be the only fact that we know about this model, while the typical set of questions related to any integrable model (the inverse scattering transform, conservation laws, explicit solutions) is still to be studied.

The (1+2)-dimensional systems (5.18) and (5.22) have been mentioned in the literature, see $[10,15,18-20,24]$. However, these papers are devoted mostly to the interrelations between various integrable models and the algebraic structures behind them. At the same time, the conservation laws or explicit solutions are, again, have not been derived yet. From this viewpoint the results presented above not only give some additional insight into the place of these equations among other integrable models but also give possibility of presenting a wide range of explicit solutions by modifying ones obtained for the DNLSH. For example, a corollary of proposition 8.3 is that it can be used to describe the dark solitons of (5.18) and (5.22). To complete this task one has to 'extract' from (8.25) and (8.29) the explicit dependence on the lowest 'times' (indicated in (5.17) and (5.21)) and to write down proper combinations of the tau-functions.

However, these questions are out of scope of this article and, to our opinion, deserve separate studies.

\section{References}

[1] V.E. Adler and A.B. Shabat, On the one class of hyperbolic systems, Symmetry, Integrability and Geometry: Methods and Applications 2 (2006) 093.

[2] H.H. Chen, Y.C. Lee and C.S. Liu, Integrability of nonlinear Hamiltonian systems by inverse scattering method. Physica Scripta 20 (1979) 490-492.

[3] A. Dimakis and F. Müller-Hoissen, From AKNS to derivative NLS hierarchies via deformations of associative products. J. Phys. A 39 (2006) 14015-14033.

[4] G.S. França, J.F. Gomes and A.H. Zimerman, The algebraic structure behind the derivative nonlinear Schrödinger equation. J. Phys. A 46 (2013) 305201. 
[5] A.S. Fokas, On a class of physically important integrable equations, Physica D 87 (1995) 145-150.

[6] V.S. Gerdjikov, M.I. Ivanov, P.P. Kulish, Quadratic bundle and nonlinear equations, Teoreticheskaya i Matematicheskaya Fizika 44 (1980) 342-357 (English translation in: Theor. Math.Phys. 44 (1980) 784-795).

[7] V.S. Gerdjikov and M.I. Ivanov, The quadratic bundle of general form and the nonlinear evolution equations. II. Hierarchies of Hamiltonian structures. Bulgarian J. Phys. 10 (1983) 130-143 (in Russian).

[8] D.J. Kaup and A.C. Newell, On the Coleman correspondence and the solution of the massive Thirring model, Lettere Al Nuovo Cimento, Series 2, 20 (1977) 325-331.

[9] D.J. Kaup and A.C. Newell, An exact solution for a derivative nonlinear Schrödinger equation. J. Math. Phys. 19 (1978) 798-801.

[10] B.G. Konopelchenko, The non-Abelian $(1+1)$-dimensional Toda lattice as the periodic fixed point of the Laplace transform for (2+1)-dimensional integrable systems. Phys. Lett. A 156 (1991) 221-222.

[11] E.A. Kuznetsov and A.V. Mikhailov, On the complete integrability of the two-dimensional classical Thirring model, Teoreticheskaya i Matematicheskaya Fizika 30 (1977) 303-314 (English translation in: Theor. Math. Phys. 30 (1977) 193-200).

[12] J. Lenells, Exactly solvable model for nonlinear pulse propagation in optical fibers. Studies in Applied Mathematics 123 (2009) 215-232.

[13] J. Lenells and A.S. Fokas, On a novel integrable generalization of the nonlinear Schrödinger equation. Nonlinearity 22 (2009) 11-27.

[14] A.V. Mikhailov, Integrability of the two-dimensional Thirring model. Pisma v ZhETF 23 (1976) 356358 (English translation in: JETP Letters 23 (1976) 320-323).

[15] A.V. Mikhailov and R.I. Yamilov, On integrable two-dimensional generalizations of nonlinear Schrodinger type equations, Phys. Lett. A 230 (1997) 295-300.

[16] H.C. Morris, The massive Thirring model connection, J. Phys. A 12 (1979) 131-134.

[17] F.W. Nijhoff, H.W. Capel, G.R.W. Quispel, J. van der Linden, The derivative nonlinear Schrödinger equation and the massive Thirring model. Phys. Lett. A 93 (1983) 455-458.

[18] A.B. Shabat and R.I. Yamilov, To a transformation theory of two-dimensional integrable systems, Phys. Lett. A 227 (1997) 15-23.

[19] I.A.B. Strachan, A new family of integrable models in (2+1) dimensions associated with Hermitian symmetric spaces, J. Math. Phys. 33 (1992) 2477-2482.

[20] T. Tsuchida, Systematic method of generating new integrable systems via inverse Miura maps, J. Math. Phys. 52 (2011) 053503.

[21] V.E. Vekslerchik, Functional representation of the Ablowitz-Ladik hierarchy. II. J. Nonlin. Math. Phys. 9 (2002) $157-180$.

[22] V.E. Vekslerchik, Bäcklund transformations between the AKNS and DNLS hierarchies, J. Phys. A 44 (2011) 465207.

[23] V.E. Vekslerchik, Functional representation of the negative AKNS hierarchy, J. Nonlin. Math. Phys. 19 (2012) 353-372.

[24] Yufeng Zhang, A (2+1)-dimensional integrable hierarchy and its extending integrable model. Chaos Solitons and Fractals 27 (2006) 555-559.

\section{Appendix A. Proof of Proposition}

The proof is straightforward (but rather tedious) application of (3.7), (5.7) together with (3.1), (5.1). Below we outline the main steps leaving the details omitted.

First, starting from (3.7) one can derive

$$
i \partial(\xi) \mathscr{J}(\zeta)=\zeta^{-1}\left[H(\xi)-\mathbb{T}_{\zeta} H(\xi)\right] G(\zeta)
$$

that can be rewritten, with the help of (3.1), as

$$
i(\xi-\zeta) \partial(\xi) \mathscr{J}(\zeta)=H(\xi) A(\xi, \zeta) B(\xi, \zeta)
$$


where

$$
\begin{aligned}
& A(\xi, \zeta)=\xi G(\zeta)-\zeta G(\xi), \\
& B(\xi, \zeta)=\left(\mathbb{T}_{\xi \zeta} U\right) V-\left(\mathbb{T}_{\zeta} U\right)\left(\mathbb{S}_{\xi} V\right)
\end{aligned}
$$

On the other hand, application of (3.3) to the derivative of (6.7) gives

$$
i \xi \partial_{1} \mathscr{K}(\zeta, \xi)=-A(\xi, \zeta) B(\xi, \zeta) H(\xi)
$$

which proves (6.6a).

Considering (6.6b), its verification is based on the identity

$$
\left[1-\zeta \eta\left(\mathbb{T}_{\zeta} U\right)\left(\overline{\mathbb{S}}_{\eta} v\right)\right] h(\eta)=\left[1-\zeta \eta\left(\mathbb{T}_{\zeta} \overline{\mathbb{T}}_{\eta} u\right) V\right]\left(\mathbb{T}_{\zeta} h(\eta)\right)
$$

which is, again, a consequence of (5.1). Differentiating (6.5) and using (A.6) one can obtain an equation, which is similar to (A.1):

$$
i \bar{\partial}(\eta) \mathscr{J}(\zeta)=\zeta^{-1}\left[\mathbb{T}_{\zeta} h(\eta)-h(\eta)\right] G(\zeta) .
$$

On the other hand, the expression for derivative $\partial_{1} \mathscr{L}$, that stems from the $\xi \rightarrow 0$ limit of (5.7c), (5.7d) combined with (3.3),

$$
i \partial_{1} \mathscr{L}(\zeta, \eta)=\eta\left[1-\left(\mathbb{T}_{\zeta} U\right)\left(\overline{\mathbb{S}}_{\eta} v\right)\right] G(\zeta) h(\eta)-\eta\left[1-\left(\overline{\mathbb{T}}_{\eta} u\right) V\right] \mathscr{L}(\zeta, \eta)
$$

can be transformed, using again (A.6), into

$$
i \partial_{1} \mathscr{L}(\zeta, \eta)=\eta\left[1-\left(\mathbb{T}_{\zeta} U\right)\left(\overline{\mathbb{S}}_{\eta} v\right)\right] G(\zeta) h(\eta)-\eta\left[1-\left(\mathbb{T}_{\zeta} \overline{\mathbb{T}}_{\eta} u\right) V\right] G(\zeta)\left(\mathbb{T}_{\zeta} h(\eta)\right)
$$

from which equation (6.6b) follows immediately. 